\title{
Thermal limiting effects in optical plasmonic waveguides
}

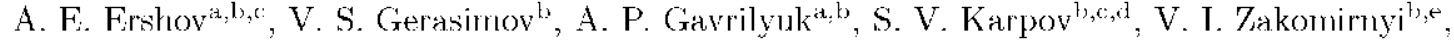 \\ I. L. Rasskazov ${ }^{\mathrm{f}, *}$, S. P. Polyutov ${ }^{\mathrm{b}}$ \\ "Institule of Conpulational Modeliny, Federul Rescarch Center KSCSB RAS, 660036, Krusnoyarsk, Russiu \\ ${ }^{b}$ Institute of Nanotechnology, Spertroscopy and Quantum Chemistry, Siberian Federal Unimersity, Krasnoyarsk, 660041, \\ Russia \\ "Siberian State Aerospace University. 660037, Krasnoyarsh, Russia \\ ${ }^{d}$ Kirensky Institute of Physirs, Fedewt Reseawh Center KSC SB RAS, G60036, Krasnoyarsk. Russin \\ ERoyal lnstitute of Technology, Stockholm SE-100 仵, Sweden \\ ${ }^{f}$ The Beckman Institule for Advanced Scicnece and Technology. University of Ilbinois at Unbance-Chartpuign. \\ Urbara, Illinois 61801, USA
}

\begin{abstract}
We have studied thermal effects occurring during excitation of optical plasmonic waveguide (OPW) in the form of linear chain of spherical Ag nanoparticles by pulsed laser radiation. It was shown that heating and subsequent melting of the first irradiated particle in a chain can significantly deteriorate the transmission efficiency of $\mathrm{OP} W$ that is the crucial and limiting factor and continuous operation of $\mathrm{OP} W$ requires cooling devies. This effect is caused by suppression of particle's surface plasmon resonance due to reaching the melting point temperature. Wo have determined optimal excitation parameters which do not significantly aflect the transmission efficiency of $\mathrm{OPW}$.
\end{abstract}

Keyuords: plasmon resonance, optical plasmonic waveguide, surface plasmon polariton, thermal effects

\section{Introduction}

Optical plasmonic waveguides (OPW) in the form of plasmonic nanoparticle chains [1 5$]$ attract significant attention in recent years due to their applicability to transmit modulated and spatiallylocalized optical radiation by means of surfaceplasmon polaritons (SI'P's). 'T'his interest is associated with vast majority of applications of such chains, particularly in sensing [6-9], nanoantemas $[10,11]$ or creation of nanoscale opti-

\footnotetext{
* Corresponding author

Fmal addresses: karpov@iph.krasn.ru
}

(S. V. Karpov), il .rasskazov@gmail. com (I. L. Rasskazov) cal integrated functional elements for new types of computing devices operating at optical frequencies at a scales much smaller than the radiation wavelength $\left[\begin{array}{ll}12 & 14\end{array}\right]$ and so on.

'Ihe influence of disorder [15-17], waveguide geometry [18-20], particles' shape [21-24] on OPW optical propertics were investigated in details in recent years. The effect of a dielectric or metallic substrate on transmission $[25,26]$ and dispersion $[27,28]$ properties of OPW which is of primary importance for practical application of plasmonic chains has been also considered. In most studies, the only one, for example, the leftrnost 
(first) nanoparticle in the chain is excited by external radiation $[15-17,25,26]$. Obviously, such excitation will be accompanied by heating of both the particles and surrounding medium. Heating of nanoparticles by laser radiation was studied in recent works [29-31]. However, these studies did not take into account the effect of heating on the optical properties of nanoparticles and vice versa. Earlier, in Ref. [32], the model of optodynamical effects in resonant domains of plasmonic nanoparticle aggregates was presented. This model takes into account light-induced changes of inter-particle forces as well as the permittivity of a particle material which occur in high intensity pulsed laser radiation. Further studies made it possible to consider the effect of heating of the particle metal core on the quality factor of surface plasmon resonance and to develop the improved version of the optodynamical model $[33,34]$. It was shown that heating of particles and subsequent melting significantly affect their resonant properties. Therefore, we can conclude that such complementary phenomenon will obviously affect the transmission properties of the OPW.

The approach to the description of heat exchange between nanoparticles and the environment $[32,33,35]$ is also applicable to the case of a single laser pulse with a duration much shorter than the thermodynamic equilibrium establishing time. However, these models disregard the effect of substrate on OPW which may play the role of the cooling device. Therefore, in our work, we have developed the thermodynamic model which takes into account heat exchange between OPW nanoparticle, surrounding environment and substrate as well as

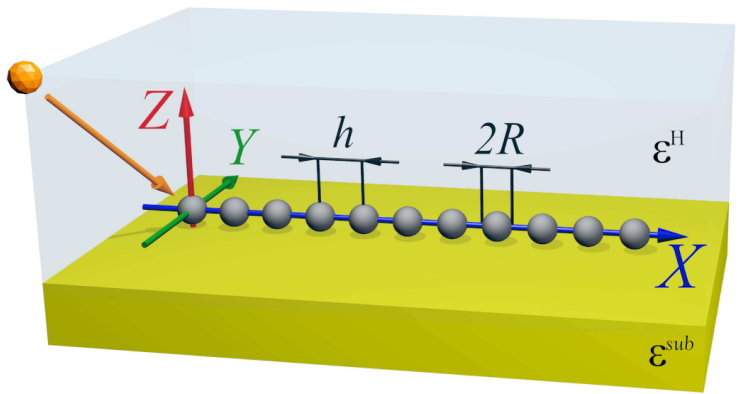

Figure 1: Schematic illustration of the of OPW geometry used in numerical simulations.

the temperature dependence of the particle optical properties.

To the best of our knowledge, there is a lack of data on the effect of heating of nanoparticles in OPW on its transmission properties. Therefore, the goal of our paper is to study the changes of OPW transmission properties associated with heating and melting of nanoparticles comprising the chain.

\section{Model}

Consider the simplest case of a linear chain consisting of $N=11$ identical spherical Ag nanoparticles with a radius $R=8 \mathrm{~nm}$. Nanoparticles are arranged equidistantly in the medium with $\varepsilon^{\mathrm{H}}=1.78$ over the surface of the quartz substrate (see Fig. 1). The distance between the centers of the neighboring particles is $h=24 \mathrm{~nm}$. The gap between 1OPW surface and substrate is set to $0.5 \mathrm{~nm}$. The particle size is assumed to be much smaller than the wavelength of incident radiation $R \ll \lambda$ and $h \leq 3 R$. Therefore, selected geometrical parameters provide the validity of the dipole approximation [15-17, 2123 ] which will be used in our calculations. 


\subsection{Electromagnetic interactions in the $O P W$}

We consider a local excitation of the OPW particles, which can be experimentally implemented by using, for example, a near-field optical microscope tip. The external field $\mathbf{E}_{n}=\mathbf{E}\left(\mathbf{r}_{n}\right)$ applied to the $n$th particle located at $\mathbf{r}_{n}$ can be described as follows:

$$
\mathbf{E}_{n}=\mathbf{E}_{0} \exp \left(i \mathbf{k r}_{n}\right) .
$$

Here $|\mathbf{k}|=2 \pi \sqrt{\varepsilon^{\mathrm{H}}} / \lambda$ is the wave vector, $\mathbf{E}_{0}$ is the amplitude of the electric component of the electromagnetic field.

Assume that the only $n=1$ nanoparticle interacts with an external field so that $\mathbf{E}_{n}=0$ for $n \neq 1$. In this case, the dipole moment $\mathbf{d}_{n}$ induced on the $n$th particle can be described by the coupled dipole approximation:

$$
\mathbf{d}_{n}=\varepsilon_{0} \alpha_{n}\left[\mathbf{E}_{n} \delta_{n 1}+\sum_{m=1}^{N} \hat{\mathbf{G}}\left(\omega ; \mathbf{r}_{n}, \mathbf{r}_{m}\right) \mathbf{d}_{m}\right],
$$

where $\alpha_{n}$ is the dipole polarizability of the $n$th particle, $\delta_{n 1}$ is Kronecker delta, $\hat{\mathbf{G}}\left(\omega ; \mathbf{r}_{n}, \mathbf{r}_{m}\right)$ is the $3 \times 3$ Green's interaction tensor describing the electric field produced at $\mathbf{r}_{n}$ by electric dipole (oscillating with frequency $\omega$ ) located at $\mathbf{r}_{m}$. For OPW on substrate, the Green's interaction tensor is defined as follows:

$\hat{\mathbf{G}}\left(\omega ; \mathbf{r}_{n}, \mathbf{r}_{m}\right)=\hat{\mathbf{G}}_{\text {free }}\left(\omega ; \mathbf{r}_{n}, \mathbf{r}_{m}\right)+\hat{\mathbf{G}}_{\text {refl }}\left(\omega ; \mathbf{r}_{n}, \mathbf{r}_{m}\right)$,

where $\hat{\mathbf{G}}_{\text {free }}\left(\omega ; \mathbf{r}_{n}, \mathbf{r}_{m}\right)$ and $\hat{\mathbf{G}}_{\text {refl }}\left(\omega ; \mathbf{r}_{n}, \mathbf{r}_{m}\right)$ are Green's tensors that describe electric field in a homogeneous environment and reflected from the substrate, correspondingly. Expression for
$\hat{\mathbf{G}}_{\text {free }}\left(\omega ; \mathbf{r}_{n}, \mathbf{r}_{m}\right)$ is well-known and can be found elsewhere. Tensor $\hat{\mathbf{G}}_{\text {refl }}\left(\omega ; \mathbf{r}_{n}, \mathbf{r}_{m}\right)$ can be written in the form of a Fourier integral [36-38]. Calculation of this integral represents quite complicated task. In some cases, the integrals of $\hat{\mathbf{G}}_{\text {refl }}\left(\omega ; \mathbf{r}_{n}, \mathbf{r}_{m}\right)$ can be evaluated along an appropriate integration path using a Gauss-Kronrod quadrature [39] or via analytical short-distance expansions [40]. However in this work, we use a purely numerical approach to compute $\hat{\mathbf{G}}_{\text {refl }}\left(\omega ; \mathbf{r}_{n}, \mathbf{r}_{m}\right)$. Specifically, we compute the Fourier integral numerically by discretization. Finally, it should be noticed that summation in (2) runs over all indices, but the term $\hat{\mathbf{G}}_{\text {free }}\left(\omega ; \mathbf{r}_{n}, \mathbf{r}_{n}\right)=0$ by definition.

An expression for the dipole polarizability for $n$th nanoparticle in homogeneous environment, which takes into account the radiation reaction correction, has the form [41]:

$$
\alpha_{n}^{-1}=\left[\alpha_{n}^{(0)}\right]^{-1}-\frac{i}{6 \pi}|\mathbf{k}|^{3},
$$

where $\alpha_{n}^{(0)}$ is so-called bare or Lorenz-Lorentz quasistatic polarizability of the particle. Selfinteraction of $n$th nanoparticle with field reflected from substrate is described by $\hat{\mathbf{G}}_{\text {refl }}\left(\omega ; \mathbf{r}_{n}, \mathbf{r}_{n}\right) \neq 0$.

In our work, we take into consideration the fact that a particle can be in the melting process. Thus, the nanoparticle is considered as a core-shell nanosphere with a solid core and a liquid shell. Therefore, the $\alpha_{n}^{(0)}$ can be described as follows [42]:

$$
\begin{aligned}
& \alpha_{n}^{(0)}=4 \pi R_{n}^{3} \\
& \times \frac{\left(\varepsilon_{n}^{\mathrm{L}}-\varepsilon^{\mathrm{H}}\right)\left(\varepsilon_{n}^{\mathrm{S}}+2 \varepsilon_{n}^{\mathrm{L}}\right)+f_{n}\left(\varepsilon_{n}^{\mathrm{S}}-\varepsilon_{n}^{\mathrm{L}}\right)\left(\varepsilon^{\mathrm{H}}+2 \varepsilon_{n}^{\mathrm{L}}\right)}{\left(\varepsilon_{n}^{\mathrm{L}}+2 \varepsilon^{\mathrm{H}}\right)\left(\varepsilon_{n}^{\mathrm{S}}+2 \varepsilon_{n}^{\mathrm{L}}\right)+2 f_{n}\left(\varepsilon_{n}^{\mathrm{S}}-\varepsilon_{n}^{\mathrm{L}}\right)\left(\varepsilon_{n}^{\mathrm{L}}-\varepsilon^{\mathrm{H}}\right)} .
\end{aligned}
$$


Here $R_{n}$ is radius of the $n$th particle, $\varepsilon_{n}^{\mathrm{S}}$ and $\varepsilon_{n}^{\mathrm{L}}$ are the temperature and size dependent permittivities of the particle material in the solid and liquid state, respectively, $f_{n}$ is the mass fraction of solid material in the particle. The expression (5) takes into account the extreme cases of completely solid $\left(f_{n}=0\right)$ and fully liquid particles $\left(f_{n}=1\right)$. The full description of $f_{n}$ will be given below. It should be noticed that $\mathrm{Ag}$ nanoparticle keeps its spherical shape even in the fully liquid state due to extremely high values of surface tension. Therefore, the expression (5) is valid for any values of $f_{n}$.

Permittivities $\varepsilon_{n}^{\mathrm{S}}$ and $\varepsilon_{n}^{\mathrm{L}}$ take into account finite size effects [43]:

$$
\varepsilon_{n}^{\mathrm{S}, \mathrm{L}}=\varepsilon_{\mathrm{tab}}^{\mathrm{S}, \mathrm{L}}+\frac{\omega_{\mathrm{pl}}^{2}}{\omega\left(\omega+i \Gamma_{0}\right)}-\frac{\omega_{\mathrm{pl}}^{2}}{\omega\left(\omega+i \Gamma_{n}\right)},
$$

where $\omega$ is the electromagnetic field frequency, $\varepsilon_{n}^{\mathrm{S}, \mathrm{L}}$ is the permittivity of the solid or liquid material of the particles, $\varepsilon_{\text {tab }}^{\mathrm{S}, \mathrm{L}}$ is the corresponding tabulated experimental values for bulk at the temperature of $300 \mathrm{~K}$ for solid material [44] and at the melting point for liquid one [45], $\Gamma_{0}$ is the time dependent bulk electron relaxation constant for corresponding temperature, $\omega_{\mathrm{pl}}$ is the plasma frequency of the particle material, $\Gamma_{n}$ is the temperature and size dependent electron relaxation constant of a particle:

$$
\Gamma_{n}=\Gamma_{0}\left(T_{n}^{\mathrm{ion}}\right)+A \frac{\mathrm{v}_{\mathrm{F}}}{R_{n}} .
$$

Here $v_{F}$ is the Fermi velocity. The value of $A$ is taken to be unit in most of cases [42]. However, the relaxation processes depend on the state of particle surface and on other factors. The dependence of the relaxation constant for bulk material $\Gamma_{0}(T)$ on the temperature can be approximated by the following expression [46]:

$$
\Gamma_{0}(T)=b T+c
$$

where $b$ and $c$ are the linear dependence coefficients obtained by linear approximation of the experimental data [47]. Detailed analysis of this model will be published elsewhere.

To describe the transmission properties of OPW we use the transmission coefficient $[15,20,22]$ :

$$
Q_{\mathrm{tr}}=\frac{\left|\mathbf{d}_{N}(t)\right|}{\left|\mathbf{d}_{1}(t=0)\right|},
$$

where $\mathbf{d}_{N}(t)$ and $\mathbf{d}_{1}(t=0)$ are oscillation amplitudes of dipole moments induced on the last (at arbitrary moment of time) and the first (at initial moment of time) particles, correspondingly.

\subsection{Thermal effects in $O P W$}

Two-component model has been developed to describe thermodynamic processes associated with heating of $\mathrm{Ag}$ nanoparticles and their environment. Model separately calculates the temperature of the ion and electron components of the particle. The temperature of the electron subsystem $T_{n}^{\mathrm{e}}$ which depends on the absorption of radiation energy by nanoparticle and on heat exchange with the crystal lattice (ion subsystem) is described by the following expression [32]:

$$
C_{n}^{\mathrm{e}} \frac{d T_{n}^{\mathrm{e}}}{d t}=-g\left[T_{n}^{\mathrm{e}}-T_{n}^{\mathrm{ion}}\right]+\frac{W_{n}}{V_{n}},
$$

where $T_{n}^{\text {ion }}$ is the temperature of ion component, $C_{n}^{\mathrm{e}}=68 T_{n}^{\mathrm{e}} \mathrm{J} \cdot \mathrm{m}^{-3} \cdot \mathrm{K}^{-1}$ is the volumetric heat capacity, $V_{n}$ is the volume of a spherical particle, $g=4 \times 10^{16} \mathrm{~J} \cdot \mathrm{m}^{-3} \cdot \mathrm{K}^{-1}$ is the energy exchange 
rate between electron and ion subsystems, $W_{n}$ is the radiation power absorbed by the particle. In dipole approximation, $W_{n}$ can be found using the equation [33]:

$$
W_{n}=\frac{\omega\left|\mathbf{d}_{\mathbf{n}}\right|^{2}}{2 \varepsilon_{0}} \operatorname{Im}\left(\frac{1}{\alpha_{n}^{*}}\right),
$$

where asterisk denotes the complex conjugate.

The developed model takes into account the phase transition at the melting point of a particle. Therefore, the heat exchange between ion component of the particle with its electronic component and the environment is described in terms of the amount of heat $Q_{n}^{\text {ion }}$ which is absorbed by a particle [32]:

$$
\frac{d Q_{n}^{\mathrm{ion}}}{d t}=g V_{n}\left[T_{n}^{\mathrm{e}}-T_{n}^{\mathrm{ion}}\right]+v_{n}
$$

where $v_{n}$ is the rate of heat transfer between a particle and its environment. The temperature of the ion subsystem of a particle during the melting process is described as follows [33]:

$T_{n}^{\text {ion }}= \begin{cases}\frac{Q_{n}^{\text {ion }}}{C_{n}^{\text {ion }} V_{n}}, & \text { when } Q_{n}^{\text {ion }}<Q_{n}^{(1)} \\ T_{n}^{\mathrm{L}}, & \text { when } Q_{n}^{(1)} \leq Q_{n}^{\text {ion }} \leq Q_{n}^{(2)} \\ \frac{Q_{n}^{\text {ion }}-L V_{n}}{C_{n}^{\text {ion }} V_{n}}, & \text { when } Q_{n}^{\text {ion }}>Q_{n}^{(2)} .\end{cases}$

Here $L$ is the volumetric heat of fusion for silver, $C_{n}^{\text {ion }}$ is volumetric heat capacity of the ion subsystem of the $n$th particle material, $T_{n}^{\mathrm{L}}=T^{\mathrm{L}}\left(R_{n}\right)$ is the size dependent melting temperature [48], $Q_{n}^{(1)}$ is the heat that corresponds to the onset of the particle melting:

$$
Q_{n}^{(1)}=C_{n}^{\mathrm{ion}} V_{n} T_{n}^{\mathrm{L}}
$$

and $Q_{n}^{(2)}$ is the heat that corresponds to the end of particle's melting process:

$$
Q_{n}^{(2)}=Q_{n}^{(1)}+L V_{n}
$$

Thus, we can determine the mass fraction $f_{n}$ of the molten material in equation (5) as follows:

$$
f_{n}= \begin{cases}0, & \text { when } Q_{n}^{\text {ion }}<Q_{n}^{(1)} \\ \frac{Q_{n}^{\text {ion }}-Q_{n}^{(1)}}{C_{n}^{\text {ion }} V_{n}}, & \text { when } Q_{n}^{(1)} \leq Q_{n}^{\text {ion }} \leq Q_{n}^{(2)} \\ 1, & \text { when } Q_{n}^{\text {ion }}>Q_{n}^{(2)}\end{cases}
$$

The rate of heat transfer from a particle to the environment can be found using the following expression [49]:

$$
v_{n}=-\varkappa \int_{S_{n}} \nabla T(\mathbf{r}, t) \cdot \mathbf{n} d S .
$$

Here $\varkappa$ and $T(\mathbf{r}, t)$ are coefficients of thermal conductivity and the temperature of host medium, correspondingly, and $\mathbf{n}$ is the normal vector to the particle surface. The integration is performed over the surface $S_{n}$ of the $n$th particle. The rate of radiative heat exchange is much less than the rate of heat transfer due to the thermal conductivity so that the first factor is omitted in this model.

The heat equation for the environment surrounding a particle is solved to determine the values of $T(\mathbf{r}, t)$ :

$$
\frac{\partial T(\mathbf{r}, t)}{\partial t}=a \Delta T(\mathbf{r}, t),
$$

where $a$ is the thermal diffusivity of the environment material. The following initial and boundary conditions are set: 
- the temperatures of a particle and surrounding medium are equal at the initial moment of time $t=0: T_{n}^{\text {ion }}=T_{n}^{\mathrm{e}}=T(\mathbf{r}, t=0)=T_{0}=300 \mathrm{~K} ;$

- the temperature of the environment at the surface of the constant-temperature substrate is time-independent: $T\left(\mathbf{r}_{\mathrm{sub}}, t\right)=T_{0}=300 \mathrm{~K}$;

- the temperature of environment is constant at infinite distance i.e. at $|\mathbf{r}| \gg \max _{n}\left(R_{n}\right): T(x=$ $\pm \infty, y, z, t)=T(x, y= \pm \infty, z, t)=T(x, y, z=$ $+\infty, t)=300 \mathrm{~K}$;

- the temperature of surrounding media on the particle surface is equal to the temperature of the ion subsystem of a particle: $T\left(\left|\mathbf{r}-\mathbf{r}_{n}\right|=R_{n}, t\right)=T_{n}^{\text {ion }}$.

\section{Results and discussion}

Before discussing the electromagnetic interactions and thermal effects in OPW, it is insightful to investigate these phenomena for single Ag nanoparticle in the host medium with dielectric permittivity equals to the one for water. We consider the extinction efficiency $Q_{\text {ext }}$ of $\mathrm{Ag}$ sphere for different temperatures and states of matter. Fig. 2 shows that extinction efficiency drops by the factor of 1.8 and 3 when the temperature of nanoparticle reaches the melting point $(T \approx 1080 \mathrm{~K})$ and when the nanopartilce becomes fully liquid, correspondingly. However the maximum of extinction spectra keeps its position through the whole thermodynamic process. Therefore, we can conclude that spherical nanoparticle does not experience any reshaping effects, and suppression of plasmon resonance is explained only by changes of optical constants of a nanoparticle material upon the temperature variation.

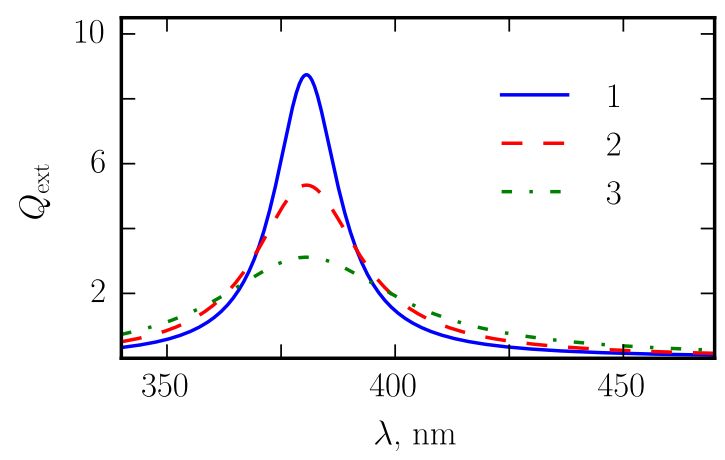

Figure 2: Extinction spectra of single Ag nanoparticle with $R=8 \mathrm{~nm}$ for different values of temperatures and states of matter: 1 - at room temperature; 2 - at the melting temperature in solid state; 3 - at the melting temperature in liquid state.

Next, we will consider the transmission properties of OPW with geometrical parameters described in Sec. 2. Fig. 3 shows transmission coefficient $Q_{\mathrm{tr}}$ for three different polarizations (aligned with Cartesian axes) of incident radiation at the initial $t=0$ moment of time when the temperature of the whole system is $T=300 \mathrm{~K}$. It is clearly seen from Fig. 3 that the maximum value of $Q_{\mathrm{tr}}$ is reached at $\lambda=402 \mathrm{~nm}$ for $\mathrm{X}$ polarization (see coordinate system on Fig. 1). Appearance of several peaks for $Q_{\operatorname{tr}}(\lambda)$ which are well pronounced for $\mathrm{X}$ and $\mathrm{Z}$ polarizations are explained by the coupling between these polarizations in the presence of substrate. This phenomenon is extensively studied in Refs. [25, 26, 28] and we do not address this effect in our work. Since X polarization provides maximum transmission efficiency, only this case will be considered in further calculations.

We now turn to the discussion of the temperature kinetics in OPW. It is obvious that thermal effects in OPW strongly depend on the intensity $I$ 


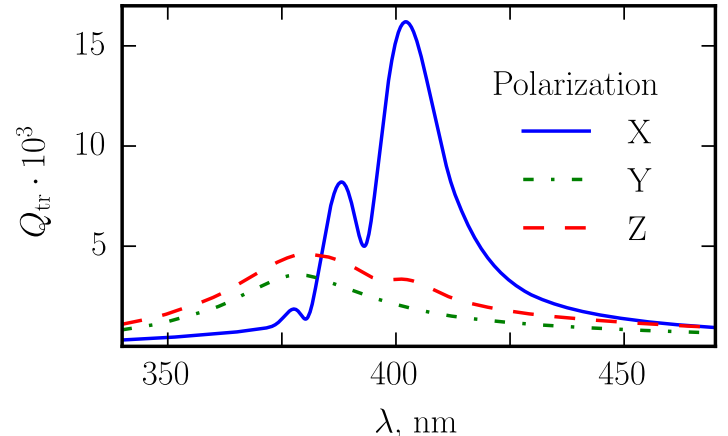

Figure 3: Transmission spectra of the OPW for different polarizations of laser radiation. Temperature of the system is $T=300 \mathrm{~K}$.

of incident laser radiation. For low values of $I$, none of nanoparticles will reach the melting point temperature. However, in this case, it will be nearly impossible to register optical signal at the terminal of OPW due to the strong SPP attenuation. In accordance with Fig. 3, SPP amplitude at the last particle of OPW drops by 70 times even in the case of the best transmission. For high values of $I$, we will observe extreme heating of OPW nanoparticles. In this case, their resonant properties will be suppressed dramatically. Therefore, in our paper we will consider the case of intermediate magnitude of intensity between these two extreme cases. For the best demonstration of nanoparticle heating and melting processes, in further calculations, we set the intensity to $I=1.57 \times 10^{8} \mathrm{~W} / \mathrm{cm}^{2}$ value which is reachable for common lasers. For these parameters of excitation, only the first excited nanoparticle reaches the melting point. We expect that in this case the OPW transmission efficiency will not drop dramatically.

Fig. 4 shows the time dependence of the ionic subsystem temperature for the first three particles in

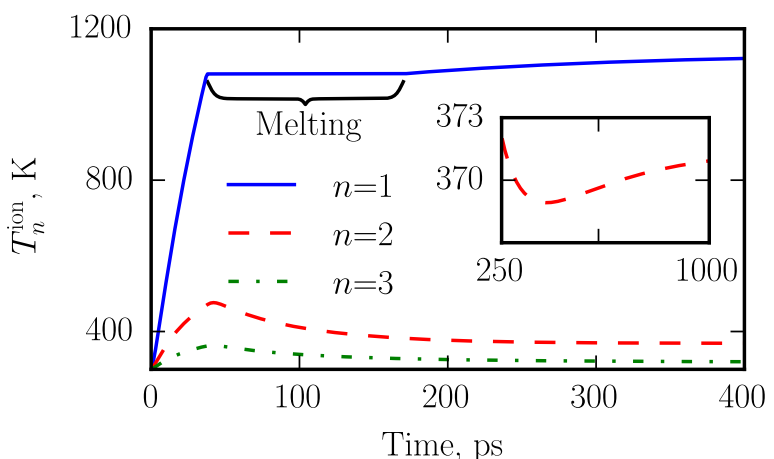

Figure 4: Temperature of ion subsystem for first, second and third OPW nanoparticles during 1 ns excitation with $\lambda=402 \mathrm{~nm}$ and intensity $I=1.57 \times 10^{8} \mathrm{~W} / \mathrm{cm}^{2}$. The inset shows the slow increase of $T_{2}^{\text {ion }}$ in $250-1000$ ps time range.

OPW when the first particle is excited by laser radiation with $\mathrm{X}$ polarization at $\lambda=402 \mathrm{~nm}$. We can see that the $n=1$ nanoparticle reaches the melting point in $37 \mathrm{ps}$. The $n=2$ and $n=3$ nanoparticles reach maximum temperatures $T_{2}^{\text {ion }} \approx 480 \mathrm{~K}$ and $T_{3}^{\text {ion }} \approx 350 \mathrm{~K}$, correspondingly, at the same moment of time. After $37 \mathrm{ps}$, for the $n=1$ nanoparticle the melting process continues during approximately 134 ps and then, its temperature slightly increases. Finally, for $t>300 \mathrm{ps}$, the temperature of the first three nanoparticles does not change longer: $T_{1}^{\text {ion }} \approx 1140 \mathrm{~K}, T_{2}^{\text {ion }} \approx 375 \mathrm{~K}$ and $T_{3}^{\text {ion }} \approx 310 \mathrm{~K}$. However, the heat from $n=1$ nanoparticle reaches $n=2$ nanoparticle at $t \approx 420 \mathrm{ps}$ and $T_{2}^{\text {ion }}$ slightly increases in the time range 500-1000 ps (see the inset in Fig. 4). The inset shows that the temperature of a second particle negligibly increases during approximately $1 \mathrm{~ns}$. $T_{n}^{\text {ion }}$ of $n \geq 4$ nanoparticles also keeps nearly unchanged for the given parameters of laser radiation.

Now we turn to consideration of the temperaturedependent transmission spectra. Fig. 5 shows the 


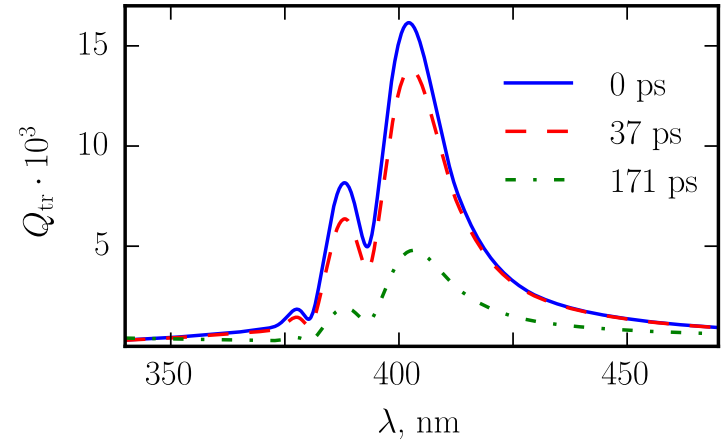

Figure 5: Transmission spectra of the OPW exposed to laser radiation with intensity $I=1.57 \times 10^{8} \mathrm{~W} / \mathrm{cm}^{2}$ at different moments of time: the initial moment of time, $t=0$ (solid line); the beginning of $n=1$ nanoparticle melting, $t=37 \mathrm{ps}$ (dashed line); the end of $n=1$ nanoparticle melting, $t=$ 171 ps (dash-dot line).

transmission spectra of OPW (expression (9)) at different stages of $n=1$ particle melting process. The OPW transmission spectrum slightly changes when the $n=1$ nanoparticle reaches the melting temperature $(T \approx 1080 \mathrm{~K}$, dash line $)$. This change is associated with the variation of the dielectric constant of the particle material. It should be noticed, that the nanoparticle is still in the solid state at 37 ps. However significant suppression of the $n=1$ nanoparticle resonant properties occurs when it becomes fully liquid at 171 ps moment of time (the end of the first nanoparticle melting process). It is clearly seen that the OPW transmission efficiency drops threefold in this case (dash-dot line). As a consequence, the transmitted energy is also reduced. In turn, this leads to the decrease of the temperature of the second and subsequent nanoparticles (see Fig. 4). The further increase of the particle temperature in a chain occurs due to heat exchange between them (see inset in Fig. 4).

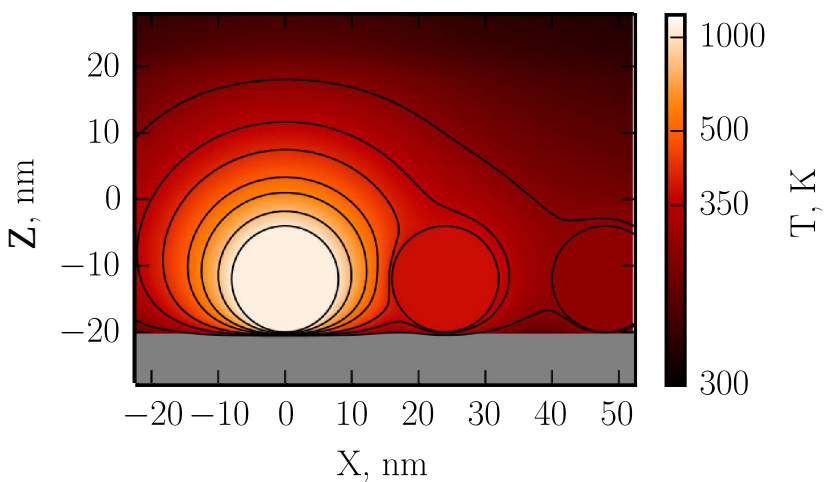

Figure 6: The temperature $T$ distribution at $t=1 \mathrm{~ns}$ for first three OPW nanoparticles at the central cross-section, $y=0$. The $n=1$ nanoparticle is exposed to pulsed laser radiation with $I=1.57 \times 10^{8} \mathrm{~W} / \mathrm{cm}^{2}$ intensity. Note that colormap is given in nonlinear scale for convenience.

Fig. 6 represents XZ-plane $(y=0)$ temperature distribution in the OPW at $t=1 \mathrm{~ns}$. The constanttemperature substrate is shown schematically. We see that only the first three particles are significantly heated, and the temperature of the $n=1$ particle reaches the melting point which is in full agreement with Fig. 4. Furthermore, the temperature distribution shows that heat transfer from the hotter particles to less heated ones takes place along the chain due to heat exchange through an environment.

In practice, OPW is assumed to be used for transmission of the digital signals, i.e. the sequence of ones and zeros representing the presence and absence of a pulse. Therefore, it is sufficient to consider the most extreme OPW excitation conditions in order to investigate limitations of OPW operating parameters and changes of its transmission properties caused by heating of nanoparticles. Thus, we will apply the excitation condi- 
tions with maximum radiation exposure of the initial nanoparticle in a chain by pulse train with zero time gap between pulses (for example, non-returnto-zero (NRZ) encoding [50]), which actually corresponds to continuous wave $(\mathrm{CW})$ excitation radiation. In this case, the heating effect in OPW is maximum. In addition, we set the condition: the OPW must deliver at least 3 photons to its terminal in order to register and to verify the transmitted signal. Thus, we will use the term "data transfer rate" to describe the frequency of registration of 3 photons at the terminal of OPW.

Therefore, it is convenient to describe the waveguide bandwidth in terms of data transfer rate which is defined as $\nu=1 / \tau$, where $\tau$ is the pulse duration. Here, the term "waveguide bandwidth" is interpreted as the ability of the waveguide to deliver optical excitation with above mentioned parameters. Calculations of the particle temperatures are performed in order to determine the maximum data transfer rate transmitted by OPW for different values of pulse duration $\tau$. In this case, the intensity of radiation is chosen so that as was mentioned earlier at least three photons reaches the OPW terminal during each pulse. It should be noticed that in order to increase $\nu$, one should increase the intensity I. Finally, the duty cycle equals $100 \%$ in all cases.

Fig. 7 shows the change of the ionic subsystem temperature $\Delta T_{n}^{\text {ion }}=T_{n}^{\text {ion }}-T_{0}^{\text {ion }}$ for different values of data transfer rate in the steady signal transmission mode $(t \rightarrow \infty)$. Here $T_{0}^{\text {ion }}=300 \mathrm{~K}$ is the initial temperature of the system. We can see that in the case of pulse duration $\tau>1.7 \times 10^{-13} \mathrm{~s}$, nanoparticles do not reach the melting point temperature. Corresponding critical intensity that pro-

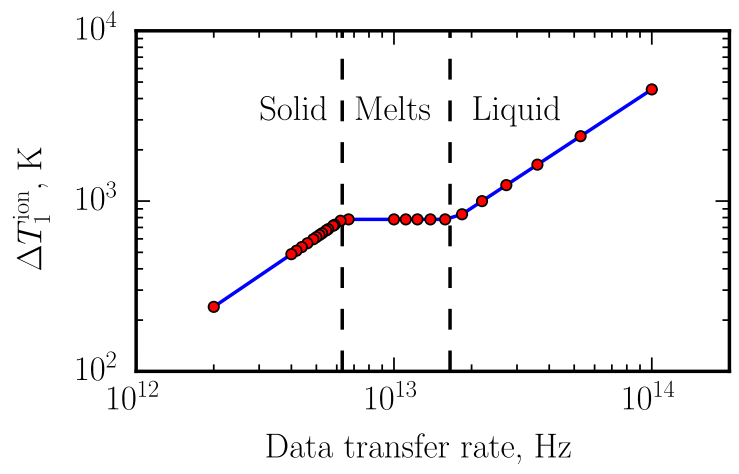

Figure 7: The temperature change of ionic subsystem for $n=1$ nanoparticle temperature change for different values of data transfer rate.

vides conditions for the onset of the melting process is $I_{\mathrm{cr}}=5.3 \times 10^{7} \mathrm{~W} / \mathrm{cm}^{2}$. Thus, the data transfer rate $\nu=6 \times 10^{12} \mathrm{~s}^{-1}$ is the optimal to maintain the transmission properties of the waveguide.

Therefore, in the practical implementation of transmission of pulsed laser radiation through the OPW one should carefully choose the intensity $I$ and the pulse duration $\tau$. All of these parameters should be selected to prevent melting of the OPW particles since this phenomenon degrades the waveguiding properties of OPW.

\section{Conclusion}

We have developed a theoretical model that describes light-induced dipole interaction between nanoparticles and surrounding medium in highintensity optical fields taking into account thermal effects. The proposed model includes the temperature dependence of dielectric permittivity of the particle material and heat exchange between nanoparticles and host medium.

The developed model was applied to investigation of an important aspect of the practical imple- 
mentation of OPW for surface plasmon polariton transmittance. This aspect is associated with thermal effects occurring during excitation of nanoparticles in OPW by pulsed laser radiation. We have shown that thermal effects significantly impair transmission efficiency of OPW due to suppression of the nanoparticle surface plasmon resonance and deterioration of its quality factor upon heating of nanoparticles and their transition to the liquid state. Optimal conditions for stable waveguiding are limited by intensity and wavelength of incident pulsed laser radiation. Another important factor which significantly affects the transmission properties of OPW is the data transfer rate. We have determined the optimal pulse repetition rate which allows one to avoid the melting of particles and therefore, to maintain continuously the transmission efficiency of OPW.

The obtained results may offer new applications of OPWs based on its high sensitivity to the intensity of incident pulsed laser radiation. The further development of the proposed model and study of thermal effects in OPWs or other periodic nanostructures may be promising within the framework of growing interest to new thermal-assisted applications in plasmonics [51].

\section{Acknowledgements}

This work was performed within the State contract of the RF Ministry of Education and Science for Siberian Federal University for scientific research in 2017-2019 and SB RAS Program No II.2P (0358-2015-0010). The numerical calculations were performed using the MVS-1000 M cluster at the Institute of Computational Modeling, Federal Research Center KSC SB Russian Academy of Sciences.

[1] M. Quinten, A. Leitner, J. R. Krenn, F. R. Ausennegg, Electromagnetic energy transport via liner chains of silver nanoparticles, Opt. Lett. 23 (17) (1998) 1331-1333.

[2] J. R. Krenn, A. Dereux, J. C. Weeber, E. Bourillot, Y. Lacroute, J. P. Goudonnet, G. Schider, W. Gotschy, A. Leitner, F. R. Aussenegg, C. Girard, Squeezing the optical near-field zone by plasmon coupling of metallic nanoparticles, Phys. Rev. Lett. 82 (1999) 2590-2593.

[3] M. L. Brongersma, J. W. Hartman, H. A. Atwater, Electromagnetic energy transfer and switching in nanoparticle chain arrays below the diffraction limit, Phys. Rev. B 62 (24) (2000) R16356-R16359.

[4] S. A. Maier, P. G. Kik, H. A. Atwater, Observation of coupled plasmon-polariton modes in Au nanoparticle chain waveguides of different length: Estimation of waveguide loss, Appl. Phys. Lett. 81 (9) (2002) 17141716.

[5] S. A. Maier, P. G. Kik, H. A. Atwater, S. Meltzer, E. Harel, B. E. Koel, A. G. Requicha, Local detection of electromagnetic energy transport below the diffraction limit in metal nanoparticle plasmon waveguide, Nature Materials 2 (2003) 229-232.

[6] M. Stewart, C. Anderton, L. Thompson, I. Maria, S. Gray, J. Rogers, R. Nuzzo, Nanostructured plasmonic sensors, Chem. Rev. 108 (2) (2008) 494-521.

[7] A. B. Evlyukhin, S. I. Bozhevolnyi, A. Pors, M. G. Nielsen, I. P. Radko, M. Willaten, O. Alberktsen, Detuned electrical dipoles for plasmonic sensing, Nano Letters 10 (2010) 4571-4577.

[8] G. Li, X. Li, M. Yang, M.-M. Chen, L.-C. Chen, X.-L. Xiong, A gold nanoparticles enhanced surface plasmon resonance immunosensor for highly sensitive detection of ischemia-modified albumin, Sensors 13 (10) (2013) 12794.

[9] V. G. Kravets, F. Schedin, R. Jalil, L. Britnell, R. V. Gorbachev, D. Ansell, B. Thackray, K. S. Novoselov, A. K. Geim, A. V. Kabashin, A. N. Grigorenko, Singular phase nano-optics in plasmonic metamaterials for label-free single-molecule detection, Nature Materials 12 (4) (2013) 304-309. 
[10] A. F. Koenderink, Plasmon nanoparticle array waveguides for single photon and single plasmon sources, Nano Letters 9 (12) (2009) 4228-4233.

[11] J. Munárriz, A. V. Malyshev, V. A. Malyshev, J. Knoester, Optical nanoantennas with tunable radiation patterns, Nano Letters 13 (2) (2013) 444-450.

[12] R. Quidant, C. Girard, J.-C. Weeber, A. Dereux, Tailoring the transmittance of integrated optical waveguides with short metallic nanoparticle chains, Phys. Rev. B 69 (2004) 085407.

[13] A. Alu, N. Engheta, Theory of linear chains of metamaterial/plasmonic particles as subdiffraction optical nanotrasmission lines, Phys. Rev. B 74 (2006) 205436.

[14] M. Février, P. Gogol, A. Aassime, R. Mégy, C. Delacour, A. Chelnokov, A. Apuzzo, S. Blaize, J.-M. Lourtioz, B. Dagens, Giant coupling effect between metal nanoparticle chain and optical waveguide, Nano Letters 12 (2) (2012) 1032-1037.

[15] V. A. Markel, A. K. Sarychev, Propagation of surface plasmons in ordered and disordered chains of metal nanospheres, Phys. Rev. B 75 (2007) 085426.

[16] F. Ruting, Plasmons in disordered nanoparticle chains: Localization and transport, Phys. Rev. B 83 (2011) 115447.

[17] I. L. Rasskazov, S. V. Karpov, V. A. Markel, Transmission and spectral properties of short optical plasmon waveguides, Opt. Spectrosc. 115 (5) (2013) 666-674.

[18] A. B. Evlyukhin, S. I. Bozhevolnyi, A. L. Stepanov, R. Kiyan, C. Reinhardt, S. Passinger, B. N. Chichkov, Focusing and directing of surface plasmon polaritons by curved chains of nanoparticles, Opt. Express 15 (25) (2007) 16667-16680.

[19] I. B. Udagedara, I. D. Rukhlenko, M. Premaratne, Surface plasmon-polariton propagation in piecewise linear chains of composite nanospheres: The role of optical gain and chain layout, Opt. Express 19 (2011) 19973.

[20] I. L. Rasskazov, S. V. Karpov, V. A. Markel, Surface plasmon polaritons in curved chains of metal nanoparticles, Phys. Rev. B 90 (7) (2014) 075405.

[21] A. A. Govyadinov, V. A. Markel, From slow to superluminal propagation: Dispersive properties of surface plasmon polaritons in linear chains of metallic nanospheroids, Phys. Rev. B 78 (3) (2008) 035403.
[22] I. L. Rasskazov, S. V. Karpov, V. A. Markel, Nondecaying surface plasmon polaritons in linear chains of silver nanospheroids, Opt. Lett. 38 (22) (2013) 4743-4746.

[23] I. L. Rasskazov, S. V. Karpov, V. A. Markel, Waveguiding properties of short linear chains of nonspherical metal nanoparticles, J. Opt. Soc. Am. B 31 (12) (2014) 2981-2989.

[24] S. Belan, S. Vergeles, Plasmon mode propagation in array of closely spaced metallic cylinders, Opt. Mat. Express 5 (1) (2015) 130-141.

[25] P. J. Compaijen, V. A. Malyshev, J. Knoester, Surfacemediated light transmission in metal nanoparticle chains, Phys. Rev. B 87 (20) (2013) 205437.

[26] I. L. Rasskazov, S. V. Karpov, G. Panasyuk, V. A. Markel, Overcoming the adverse effects of substrate on the waveguiding properties of plasmonic nanoparticle chains, J. Appl. Phys. 119 (2016) 043101.

[27] J.-W. Dong, Z.-L. Deng, Direct eigenmode analysis of plasmonic modes in metal nanoparticle chain with layered medium, Opt. Lett. 38 (13) (2013) 2244-2246.

[28] P. J. Compaijen, V. A. Malyshev, J. Knoester, Engineering plasmon dispersion relations: hybrid nanoparticle chain -substrate plasmon polaritons, Opt. Express 23 (3) (2015) 2280-2292.

[29] B. S. Luk'yanchuk, A. E. Miroshnichenko, M. I. Tribelsky, Y. S. Kivshar, A. R. Khokhlov, Paradoxes in laser heating of plasmonic nanoparticles, New Journal of Physics 14 (9) (2012) 093022.

[30] G. Baffou, P. Berto, E. B. Ureña, R. Quidant, S. Monneret, J. Polleux, H. Rigneault, Photoinduced heating of nanoparticle arrays, ACS Nano 7 (8) (2013) 64786488 .

[31] A. D. Phan, T.-L. Phan, L. M. Woods, Near-field heat transfer between gold nanoparticle arrays, Journal of Applied Physics 114 (21) (2013) 214306.

[32] A. P. Gavrilyuk, S. V. Karpov, Processes in resonant domains of metal nanoparticle aggregates and optical nonlinearity of aggregates in pulsed laser fields, Appl. Phys. B 97 (2009) 163.

[33] A. E. Ershov, A. P. Gavrilyuk, S. V. Karpov, P. N. Semina, Optodynamic phenomena in aggregates of polydisperse plasmonic nanoparticles, Appl. Phys. B 115 (4) (2014) 547-560. 
[34] V. S. Gerasimov, A. E. Ershov, A. P. Gavrilyuk, S. V. Karpov, H. Ågren, S. P. Polyutov, Suppression of surface plasmon resonance in au nanoparticles upon transition to the liquid state, Opt. Express 24 (23) (2016) 26851.

[35] S. Hashimoto, D. Werner, T. Uwada, Studies on the interaction of pulsed lasers with plasmonic gold nanoparticles toward light manipulation, heat management, and nanofabrication, Journal of Photochemistry and Photobiology C: Photochemistry Reviews 13 (1) (2012) 28 54.

[36] A. Sommerfeld, Uber die ausbreitlung der wellen in der drahtlosen telegraphie, Ann. Phys. Lpz. 28 (1909) 665736.

[37] A. A. Maradudin, D. L. Mills, Scattering and absorption of electromagnetic radiation by semi-infinite medium in the presence of surface roughness, Phys. Rev. B 11 (4) (1975) 1392-1415.

[38] W. C. Chew, Waves and fields in inhomogeneous media, Van Nostrand Reinhold, New York, 1990.

[39] M. Paulus, P. Gay-Balmaz, O. J. F. Martin, Accurate and efficient computation of the green's tensor for stratified media, Phys. Rev. E 62 (2000) 5797-5807.

[40] G. Y. Panasyuk, J. C. Schotland, V. A. Markel, Shortdistance expansion for the electromagnetic half-space green's tensor: general results and an application to radiative lifetime computations, J. Phys. A 42 (27) (2009) 275203.

[41] V. A. Markel, L. S. Muratov, M. I. Stockman, T. F. George, Theory and numerical simulation of optical properties of fractal clusters, Phys. Rev. B 43 (10) (1991) 8183-8195.

[42] C. F. Bohren, D. R. Huffman, Absorption and Scattering of Light by Small Particles, John Wiley \& Sons, New York, 1998.

[43] V. A. Markel, V. M. Shalaev, E. B. Stechel, W. Kim, R. L. Armstrong, Small-particle composites. i. linear optical properties, Phys. Rev. B 53 (5) (1996) 24252436.

[44] P. B. Johnson, R. W. Christy, Optical constants of the noble metals, Phys. Rev. B 6 (12) (1972) 4370-4379.

[45] J. C. Miller, Optical properties of liquid metals at high temperatures, Philosophical Magazine 20 (168) (1969)
$1115-1132$

[46] C. Kittel, Introduction to Solid State Physics, 6th Edition, John Wiley \& Sons, Inc., New York, 1986.

[47] M. Otter, Temperaturabhängigkeit der optischen konstanten massiver metalle, Zeitschrift für Physik 161 (5) (1961) 539-549.

[48] T. Castro, R. Reifenberger, E. Choi, R. P. Andres, Size-dependent melting temperature of individual nanometer-sized metallic clusters, Phys. Rev. B 42 (1990) 8548-8556.

[49] L. D. Landau, Theory of elasticity, ButterworthHeinemann, Oxford England Burlington, MA, 1986.

[50] S. Yao, S. Fu, H. Wang, M. Tang, P. Shum, D. Liu, Performance comparison for nrz, rz, and csrz modulation formats in rs-dbs nyquist wdm system, J. Opt. Commun. Netw. 6 (4) (2014) 355-361.

[51] J. C. Ndukaife, V. M. Shalaev, A. Boltasseva, Plasmonics - turning loss into gain, Science 351 (2016) 334-335. 